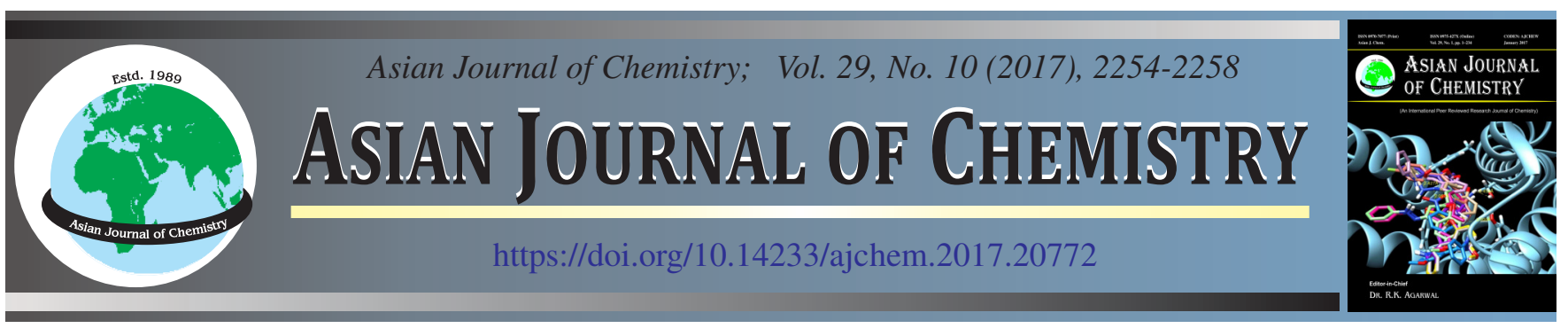

\title{
Mechanical and Structural Characterization of Eco-Friendly Films Prepared Using Poly(vinyl alcohol), Cellulose Nanocrystals and Chitosan Nanoparticle Blend
}

\section{Debomitra Dey ${ }^{1}$, S. Periyar Selvam ${ }^{1, *}$, M. Mahesh Kumar ${ }^{1}$, Emmanuel Rotimi Sadiku $^{2}$ and Anomitra Dey ${ }^{3}$}

\begin{abstract}
${ }^{1}$ Department of Food Process Engineering, School of Bioengineering, SRM University, Kattankulathur-603 203, India
${ }^{2}$ Department of Chemical, Metallurgical and Materials Engineering, Tshwane University of Technology, CSIR Campus, Lynnwood Ridge 0040, Republic of South Africa

${ }^{3}$ Institute of Chemical Technology, Mumbai-400 019, India
\end{abstract}

*Corresponding author: E-mail: periyar.india@gmail.com

\begin{abstract}
Nanocomposite films of poly(vinyl alcohol) (PVA), cellulose nanocrystals (CNC) and chitosan nanoparticles (CNP) were prepared using environmental friendly methods. These films were prepared in the concentration of 1-3\% of nanomaterials and 3-5\% PVA and the films with the highest mechanical strength was characterized by studying the morphology through SEM and intermolecular interactions using XRD. Strong intermolecular forces existed between the nanomaterials and PVA matrix, shown by the XRD results. It was observed that the addition of nanomaterials increased the tensile strength of the films. The nanocomposite films were subjected to biodegradation studies for the degree of decomposability, as the need for compostable packaging material is essential, due to the ever increasing problem of recycling of plastics. Thus creating them to be potential candidates for packaging, as they may provide high mechanical strength to maintain the integrity of the package and are biodegradable in nature.
\end{abstract}

Keywords: Cellulose nanocrystals, Environment, Biodegradable, Green materials, Chitosan nanoparticles.

\section{INTRODUCTION}

Cellulose is an abundant and naturally occurring polymer that can be obtained from numerous resources [1]. Cellulose in its native form is known to be fibrillar and crystalline [2] and the cellulose fibrils have a significant role in towards the high strength of plant cell walls [3]. The structure is organized into fibrils, which are surrounded by a matrix of lignin and hemicellulose [4,5]. The nano-sized crystals of cellulose are referred to as whiskers, nanowhiskers or nanofibrils and are obtained from sources like plant and animal [6]. The extraction of cellulose whiskers from renewable sources has gained more consideration in years to come because of its exceptional mechanical properties like tensile strength and modulus, large specific surface area, high aspect ratio, environmental benefits and comparatively lower cost [7-9].

George and Sabapathi [10] discussed that the chemical methods yield more cellulose nanocrystals (CNC) and better than mechanical method, as chemical methods $\mathrm{CNC}$ with greater crystallinity. Cellulose nanofibers are essentially widely available in environment at low costs, they are characteristically available with ease in recycling, involve low energy consumption. These attributes render them popularity for use as nanomaterials as they are low in cost, light weight and have high strength, prove to be durable, also when used at low volume.

Correspondingly, chitosan is the second most abundant polymer found in the nature. It has wide application in food and pharmaceutical industry and has been extensively studied and possess properties like biocompatibility, biodegradability, safe and non-toxic [11], thus has gained attention in the recent years in the food, textile, agriculture industries. Chitosan exhibits poor mechanical strength and strongly hydrophilic which makes it a poor candidate for food packaging [12], but the application of chitosan nanoparticle is not widely studied for packaging of food, hence, in this study we investigate the incorporation of CNP to study the improvement of mechanical and structural changes in the polymer.

It has been a well-known fact that poly(vinyl alcohol) is water soluble, biodegradable, has greater mechanical strength and is compatible with starch [13], additionally due to the above mentioned attributes it has wide application in packaging [14]. Shin et al. [15] reported that PVA provides exceptional property of gas barrier in dry state, as PVA is water soluble; it also tends to lose gas barrier properties under humid atmosphere. This is one of the limitations of using PVA in food packaging; this limitation can be curbed by the incorporation 
of nanomaterials. CNC (cellulose nanocrystal) can be used as the model nanofillers, which has adequate mechanical strength, hence can be used for blending with natural polymers like starch, chitosan and at the same time with synthetic polymers like poly(vinyl alcohol) (PVA), polypropylene [10].

The purpose of this investigation was to assess the change in mechanical and structural properties of PVA films when blended with nanomaterials like cellulose nanocrystals, chitosan nanoparticle, as they are believed to impart high mechanical strength and provide integrity to the package.

\section{EXPERIMENTAL}

Mango wastes $(10 \mathrm{~kg})$ were collected from local juice vendor in Chennai, India in the month of August 2016. Poly(vinyl alcohol) was purchased from LOBA Chemie, India. Deionized, double distilled water (Milli-Q Plus system, USA) was used for all experiments.

Preparation and characterization of chitosan nanoparticle: The chitosan nanoparticles were prepared by Calvo et al. [16] method in the ratio of 5:1 (chitosan:sodium tripolyphosphate). Tripolyphosphate was used as the cross linking agent. The preparation of nanoparticles was carried out using ionic gelation method as discussed by Nagpal et al. [17]. The characterization of the nanoparticles was done using Malvern Zeta Sizer v.7.01 (U.K.) for studying the particle size and zeta potential. All samples were prepared in triplicates using Milli Q-water.

Extraction of cellulose nanocrystals (CNC): The mango waste sample was dried in tray drier at $55^{\circ} \mathrm{C}$ and the dried samples were subjected to grounding to obtain the waste in powdered form for further use in experimental studies. These powder samples were subjected to fiber treatment using the method by Cordeiro et al. [18] and Rosa et al. [19] with slight modification. Initially, the fibers were subjected to alkaline treatment twice. First by using $2 \% \mathrm{NaOH}$ (w/v) under stirring at $80{ }^{\circ} \mathrm{C}$ for $2 \mathrm{~h}$ and this treatment was repeated once again. The fibers were washed and were dried overnight at $55^{\circ} \mathrm{C}$. Further these fibers were bleached using $30 \% \mathrm{H}_{2} \mathrm{O}_{2}$ (v/v) along with $4 \%$ $\mathrm{NaOH}(\mathrm{w} / \mathrm{v})$ under stirring at $50{ }^{\circ} \mathrm{C}$ for $3 \mathrm{~h}$ and then washed. $\mathrm{H}_{2} \mathrm{O}_{2}$ was used for bleaching as it environmental friendly as compared to other bleaching agents like sodium hypochlorite. Further, acid treatment with $52 \% \mathrm{H}_{2} \mathrm{SO}_{4}(\mathrm{w} / \mathrm{w})$ under stirring at $50{ }^{\circ} \mathrm{C}$ for $3 \mathrm{~h}$ was carried out according to Orts et al. [7] and Cranston et al. [20] in the ratio of 1:20 (fiber:solution). After hydrolysis, the reaction was dismissed with ice-cold deionized water in the ratio of 1:5 and after termination the samples were washed with deionized water five times, by centrifugation at $5000 \mathrm{rpm}$ for $15 \mathrm{~min}$, followed by ultra-sonication. The precipitate obtained by centrifugation was subjected to dialysis using dialysis bag with molecular weight cut off 12,000-14,000 (HiMedia, Mumbai, India) against water for $48 \mathrm{~h}$ until the $\mathrm{pH}$ of 7 was reached. The precipitate was diluted with deionized water to obtain the suspension of nanowhiskers and was further used for characterization and preparation of film.

Preparation of films: The films were prepared using solvent casting method, wherein water was used as the solvent and the protocol by Muppalla et al. [21] with slight modification. CNC was used in the concentration of 1-3\% and PVA was used in the concentration of 3-5\%. CNC was blended with
PVA with the above mentioned concentrations. Similarly, 1-3 \% CNP were incorporated while preparation of 3-5\% PVA films, as PVA has better film forming capacity at concentration above $3 \%$. PVA was added to lukewarm water and stirred constantly until the solution turned clear and subsequently the nanofillers CNC was added and the films were casted on petriplates; kept at room temperature overnight for drying.

Biodegradation analysis: Biodegradation is the breakdown of organic material caused by activity of microorganism through enzymatic action. For this study, biodegradation was carried out on PVA films and films blended with CNC and CNP using soil burial method in with slight modification [22]. For this study, dry soil was taken from the nursery in SRM University, India; and the study was conducted by keeping the soil in plastic box of depth $15 \mathrm{~cm}$ and the films were cut into $5 \mathrm{~cm} \times 5 \mathrm{~cm}$ pieces and buried to a depth of $8 \mathrm{~cm}$ in the box. The percentage degradation rate over the period of 30 days was recorded. Biodegradation was calculated as percentage degradation films over the period of 30 days.

Characterization of films: The films were prepared and cut into $6.5 \mathrm{~cm} \times 3 \mathrm{~cm}$ and the thickness was measured using Vernier Calliper Inder $150 \mathrm{~mm}$ and the responses of tensile strength and elongation was measured using Tensile Testing Machine TKG EC-2500 N. The films which had the highest mechanical strength were selected and further characterized for studying the morphology and structural studies. The surface morphology of cellulose nanocrystals and nanocomposite PVA films morphology were examined using by SEM (FEI Quanta FEG 200). The X-Ray diffraction analysis were performed on plain PVA films and the PVA films incorporated with nanomaterials with Panalytical B.V., Netherlands using $\mathrm{CuK}_{\alpha}$ radiation at voltage of $40 \mathrm{kV}$ and current of $45 \mathrm{~mA}$ with scanning step of $29.84 \%$ between $5^{\circ}$ to $90^{\circ}$.

\section{RESULTS AND DISCUSSION}

The results for particle size analysis is presented in Table-1, the size distribution of nanoparticles is an important parameter to study the activity and stability of the nanoparticles. The average size was observed to 390.2 and $375.7 \mathrm{~nm}$ the corresponding zeta potential was 16.5 and $16.3 \mathrm{mV}$, respectively were used for preparation of films. Prajakta et al. [23] discussed that zeta potential around $30 \mathrm{mV}$ produces homogenous and stable nanoparticles.

\begin{tabular}{ccc}
\multicolumn{4}{c}{ TABLE-1 } \\
\multicolumn{3}{c}{ RESULTS FOR PARTICLE SIZE AND ZETA } \\
POTENTIAL OF CHITOSAN NANOPARTICLE \\
\multicolumn{2}{c}{ USED FOR PREPARATION OF CNP FILMS } \\
\hline Sample ID & Particle size $(\mathrm{nm})$ & Zeta potential $(\mathrm{mV})$ \\
\hline CN1 & $390.2 \pm 1.48$ & $16.5 \pm 0.416$ \\
CN2 & $375.7 \pm 2.60$ & $16.3 \pm 1.47$ \\
\hline
\end{tabular}

It can be observed from Table-2 that mechanical properties changed with varying concentration of nanomatreials and the polymer PVA; in case $\mathrm{CNC}$, with increse in concentration both the tensile sterngth and elongation, similarly in case of incorporation of nanoparticle, this can be correlated with the finding reported by Azizi et al. [24]. The formation of hydrogen bonds formed between CNC and PVA matrix, which restricts 
motions and promotes rigidity. The $\mathrm{CNC}, \mathrm{CNP}$ and PVA films with maximum tensile strength and elongation was selected for XRD and SEM characterziation, the films selected were 2CNC/4PVA, 1CNP/3PVA and 3PVA (Table-2). It can be concluded that CNP films at lower concentration had better strength than the CNC films, with regard to the mechanical proeprties of the films. Table-2 also represents the results of biodegradation or decomposition rates of the films as a function of percentage degradation over the period of 30 days, with studies done on laboratory scale. It can be observed that the CNP films had greater rates of decomposability as compared to the $\mathrm{CNC}$ films. The percentage degradation rates depends upon the type of soil, microbiota of soil and rates of biodegradation are higher when compost is added in the soil.

The cellulose nanocrystals obtained by acid hydrolysis method were morphologically studied using SEM (Fig. 1), shows the presence of cellulose nanocrystals and the efficiency of dialysis treatment as discussed by Rosa et al. [19]. The SEM analysis was done on plain PVA and PVA films incorporated with nanomaterials CNC and CNP are presented in Figs. 2 and 3 , it can observed that the $\mathrm{CNC}$ is dispersed in the matrix of PVA, due to the presence of hydrogen bonding and electrostatic interaction [25]. Similarly the CNP films showed the presence of nanoparticle agglomerates, all along the matrix of

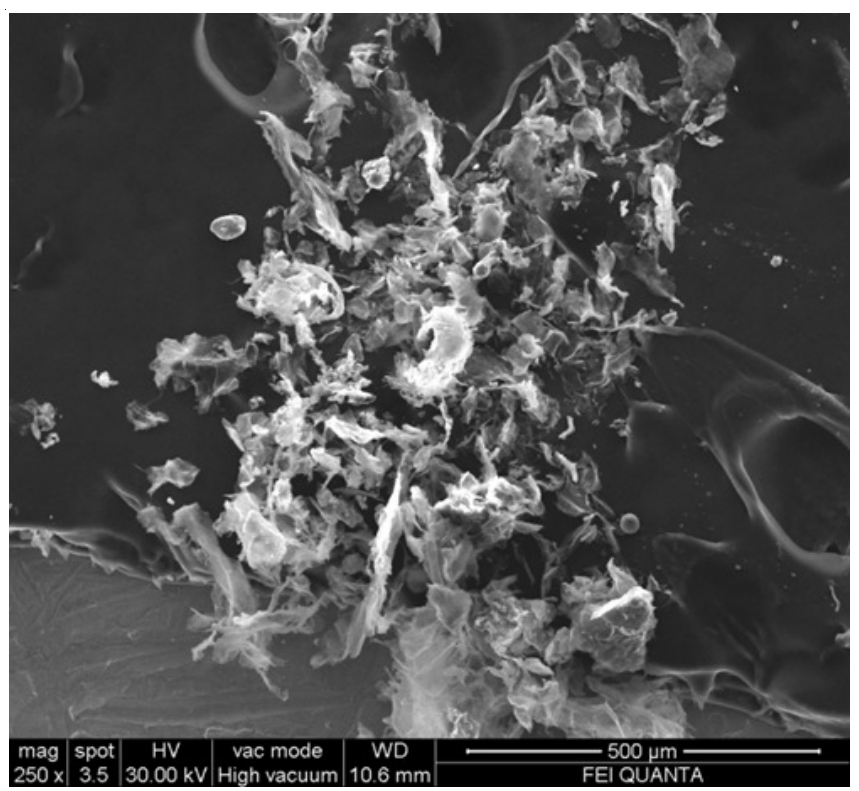

Fig. 1. SEM micrograph of cellulose nanocrystal obtained by acid hydrolysis of mango waste
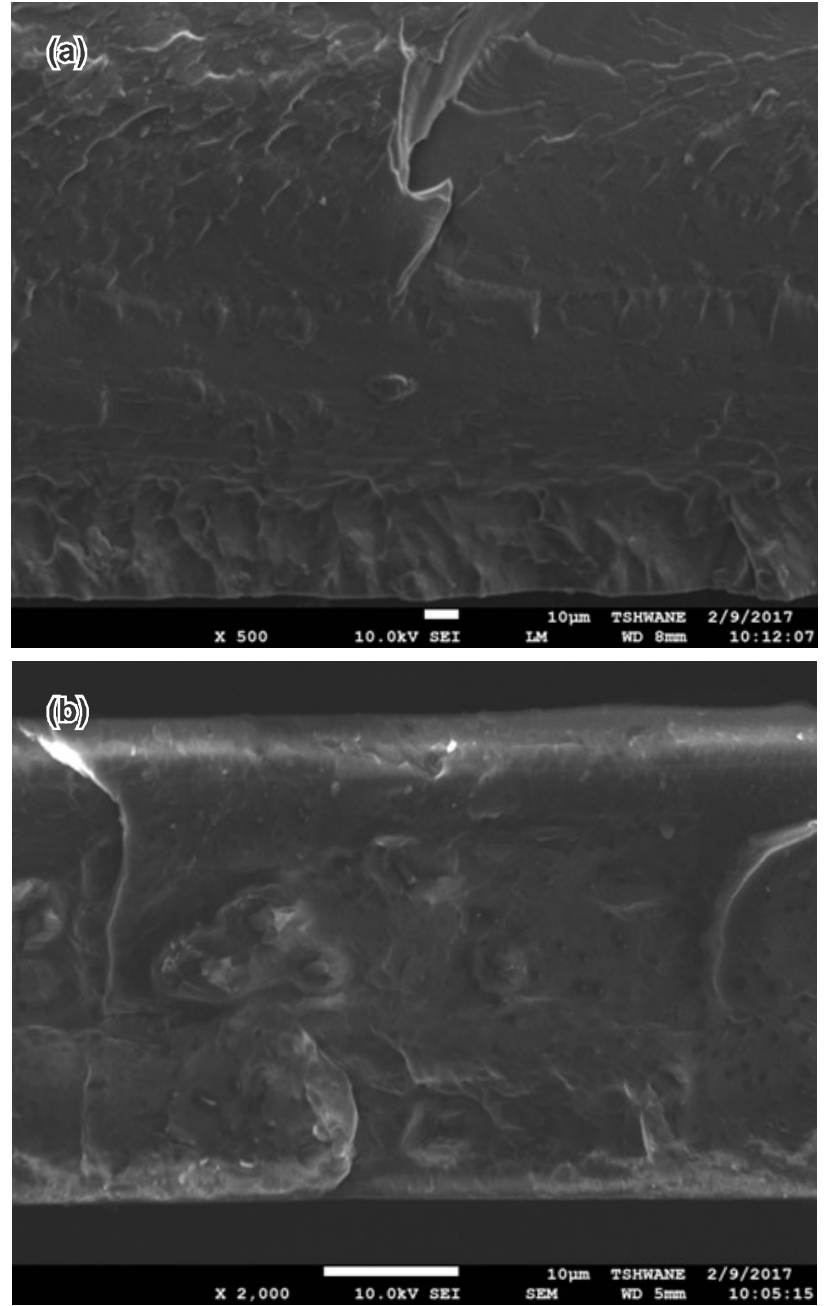

Fig. 2. SEM micrographs of (a) 4PVA/2 CNC and (b) 3PVA/1CNP

PVA due the electrostatic interaction of the ammonium groups on chitosan nanoparticles. The micrograph of PVA film presents a flat surface, with the incorporation of nanomaterials in the matrix, it is observed that rough surface implies that the nanomaterial has dispersed in the matrix.

The XRD analysis was carried out for CNC, CNP and PVA films to study the crystalline structure of the films (Figs. 4-6). The $2 \theta$ for PVA was 19.25 , with incorportaion of nanomaterials, the $2 \theta$ value decreased to $19.188^{\circ}$ and $18.99^{\circ}$ for CNC and CNP respectively (Table-3). Similar pattern has been observed by Li et al. [25] with incorporation of cellulose nanowhiskers in PVA, the $2 \theta$ was lowered. They also reproted

\begin{tabular}{ccccc}
\multicolumn{5}{c}{ TABLE-2 } \\
RESULTS FOR RESPONSES OF MECHANICAL STRENGTH viz., THICKNESS, TENSILE STRENGTH AND \\
ELONGATION; PERCENTAGE DEGRADATION OF THE FILMS OF CNC-PVA BLEND FILMS AND PVA FILMS \\
\hline Film type & Thickness (mm) & Tensile strength (MPa) & \multicolumn{1}{c}{ Elongation (\%) } & Percentage degradation (\%) \\
\hline $1 \%$ CNC + 3 \% PVA & $0.10 \pm 0.015$ & $3.639 \pm 0.110$ & $119.68 \pm 1.040$ & $64.22 \pm 1.11$ \\
$2 \%$ CNC + 4 \% PVA & $0.07 \pm 0.01$ & $5.708 \pm 0.96$ & $123.95 \pm 1.02$ & $69.49 \pm 0.001$ \\
$3 \%$ CNC + 5 \% PVA & $0.12 \pm 0.0264$ & $3.469 \pm 0.12$ & $100.51 \pm 3.729$ & $65.83 \pm 2.11$ \\
$1 \%$ CNP + 3 \% PVA & $0.066 \pm 0.023$ & $9.125 \pm 0.21$ & $122.76 \pm 3.11$ & $77.33 \pm 1.07$ \\
$2 \%$ CNP + 4 \% PVA & $0.10 \pm 0.03$ & $6.576 \pm 2.101$ & $110.62 \pm 1.728$ & $72 \pm 0.101$ \\
$3 \%$ CNP + 5 \% PVA & $0.09 \pm 0.035$ & $8.484 \pm 1.268$ & $121.53 \pm 0.58$ & $76 \pm 0.01$ \\
3\% PVA & $0.08 \pm 0.03$ & $2.668 \pm 0.481$ & $65.12 \pm 4.09$ & $55 \pm 1.131$ \\
4 \% PVA & $0.06 \pm 0.057$ & $3.397 \pm 1.511$ & $57.87 \pm 2.91$ & $53 \pm 0.173$ \\
5\% PVA & $0.07 \pm 0.015$ & $3.938 \pm 1.328$ & $53.99 \pm 2.851$ & $56 \pm 1.17$ \\
\hline
\end{tabular}

$\mathrm{CNC}=$ Cellulose nanocrystals; $\mathrm{PVA}=$ Poly $($ vinyl alcohol $)$ 


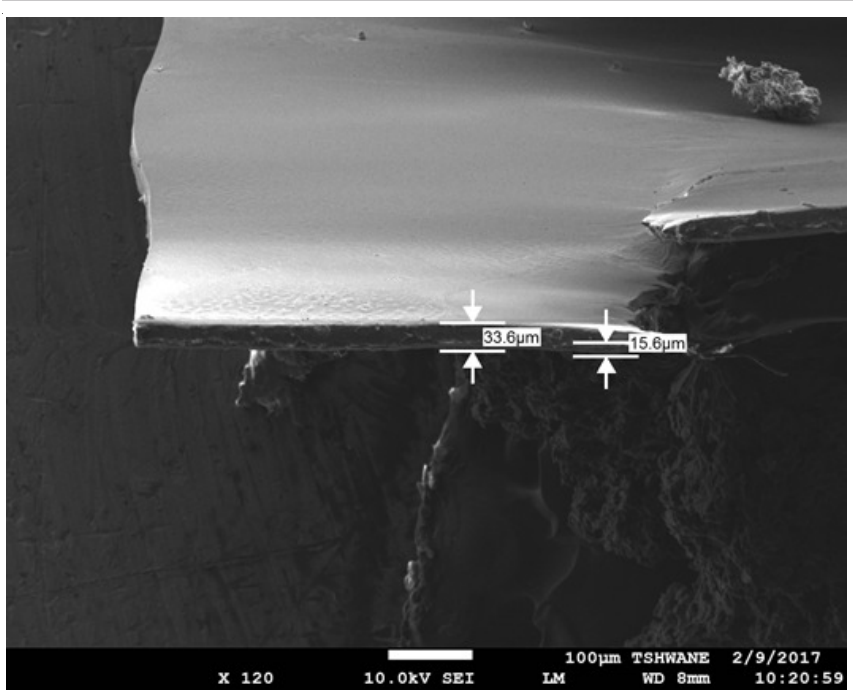

Fig. 3. SEM micrograph of $3 \%$ plain PVA film

TABLE-3

XRD DATA FOR PVA FILMS AND PVA INCORPORATED WITH NANOMATERIALS CNC AND CNP

\begin{tabular}{ccc}
\hline Specimen ID & $2 \theta\left({ }^{\circ}\right)$ & d-spacing \\
\hline 2CNC/4PVA & 19.188 & 4.621 \\
1CNP/3PVA & 18.99 & 4.66 \\
3PVA & 19.25 & 4.60 \\
\hline
\end{tabular}

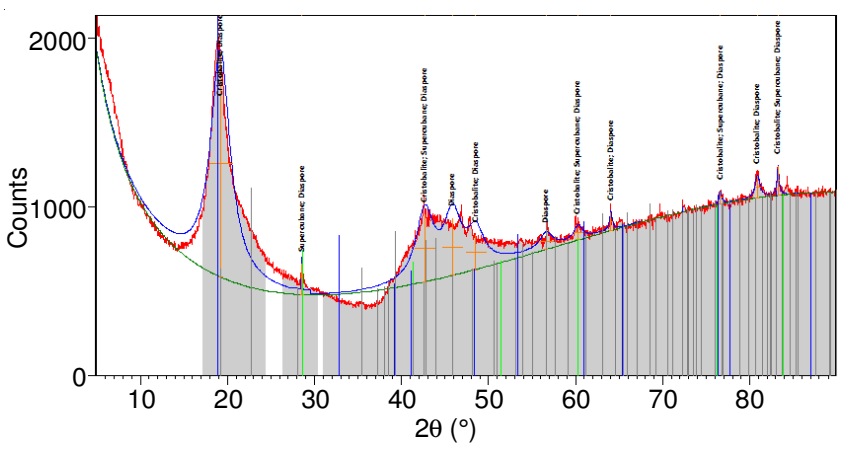

Fig. 4. XRD pattern of $4 \mathrm{PVA} / 2 \mathrm{CNC}$ film

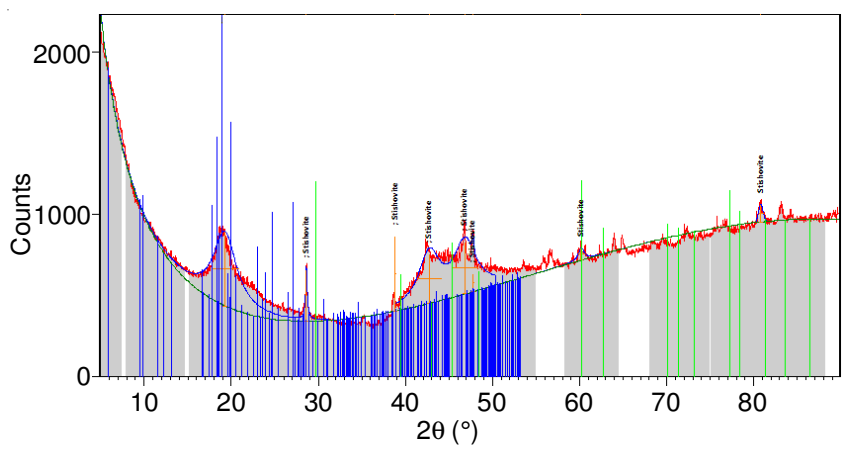

Fig. 5. XRD pattern of 3PVA/1CNP film

that with increase in chitosan content the peaks of $2 \theta$ was lowered.

Gomathi et al. [26] on XRD of chitosan nanoparticle obtained a broad peak at $18.35^{\circ}$ due to formation of chitosan nanoparticle, as comapred to chitosan which had a peak at $19.5^{\circ}$. This shift is due to the amorphous nature of cross linking of hydrogen bonds in chitosan, sodium hexaphosphate and

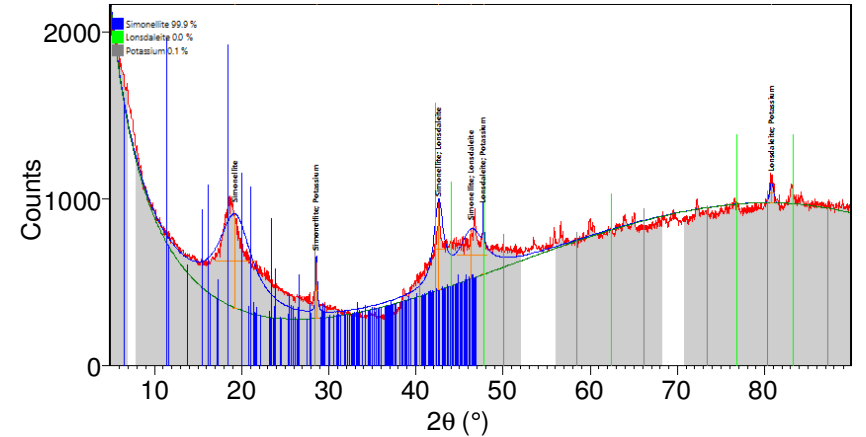

Fig. 6. XRD pattern of 3PVA film

lenalidomide used in their study. Similarly in this study we report the pattern of XRD peak for chitosan nanoparticle at $18.99^{\circ}$ and attribute to the formation of cross linking between sodium tripolyphosphate and chitosan which causes the broadening of the peaks. Hence, the greater strength of the films are attributed to the strong hydrogen bonding of nanomaterial and polymer.

\section{Conclusion}

This study validated the incorporation of CNC and CNP in PVA films by simple mixing, which can attribute to the increased mechanical strength of the films, due to intermolecular bonding between PVA matrix and the nanomaterials. These films are decomposable, as the preparation of the films has been done using eco-friendly materials. These films can be used for short term packaging of food. This focussed upon the structural and mechanical properties of the films and these films can offer endurance and integrity to the package. Hence, it is concluded that this novel packaging material can be used as a potential candidate for packaging and further studies can be conducted on the barrier properties and thermal properties of this packaging material.

\section{ACKNOWLEDGEMENTS}

This research was financially supported by the Department of Science and Technology (DST), India and the National Research Foundation (NRF), South Africa under the India and South African Bilateral Research Collaboration Program (DST/ INT/South Africa/P-07/2014). The authors express thanks to Director (Engineering and Technology) and Dean School of Bioengineering, SRM University for their cordial support. In addition, the authors express their special thanks to Dean School of Mechanical Engineering, SRM University, for providing the facilities at Strength of Materials Laboratory, Nanotechnology Research Centre (NRC) at SRM University.

\section{REFERENCES}

1. D. Klemm, B. Heublein, H.P. Fink and A. Bohn, Angew. Chem. Int. Ed., 44, 3358 (2005);

https://doi.org/10.1002/anie.200460587.

2. I.M. Saxena and R.M. Brown, Ann. Bot., 96, 9 (2005); https://doi.org/10.1093/aob/mci155.

3. R. Zuluaga, J.L. Putaux, J. Cruz, J. Velez, I. Mondragon and P. Ganan, Carbohydr. Polym., 76, 51 (2009); https://doi.org/10.1016/j.carbpol.2008.09.024

4. E.L. Hult, P.T. Larsson and T. Iversen, Cellulose, 7, 35 (2000); https://doi.org/10.1023/A:1009236932134. 
5. H.A. Krassig, Polymer Monographs, 11, 240 (1993).

6. M.A.S. Azizi Samir, F. Alloin and A. Dufresne, Biomacromolecules, 6, 612 (2005);

https://doi.org/10.1021/bm0493685.

7. W.J. Orts, J. Shey, S.H. Imam, G.M. Glenn, M.E. Guttman and J.F. Revol, J. Polymers Environ., 13, 301 (2005); https://doi.org/10.1007/s10924-005-5514-3.

8. E.S. Medeiros, L.H.C. Mattoso, E.N. Ito, K.S. Gregorski, G.H. Robertson, R.D. Offeman, D.F. Wood, W.J. Orts and S.H. Imam, J. Biobased Mater. Bioenergy, 2, 231 (2008);

https://doi.org/10.1166/jbmb.2008.411.

9. E.S. Medeiros, L.H.C. Mattoso, R. Bernardes-Filho, D.F. Wood and W.J. Orts, Colloid Polym. Sci., 286, 1265 (2008); https://doi.org/10.1007/s00396-008-1887-x.

10. J. George and S.N. Sabapathi, Nanotechnol. Sci. Appl., 8, 45 (2015); https://doi.org/10.2147/NSA.S64386.

11. P.K. Dutta, J. Dutta and V.S. Tripathi, J. Sci. Ind. Res. (India), 63, 20 (2004).

12. D. Hu, H. Wang and L. Wang, LWT-Food Sci. Technol., 65, 398 (2016); https://doi.org/10.1016/j.lwt.2015.08.033.

13. E.S.M. Negim, R.K. Rakhmetullayeva, G.Z. Yeligbayeva, P.I. Urkimbaeva, S.T. Primzharova, D.B. Kaldybekov, J.M. Khatib, G.A. Mun and W. Craig, Int. J. Basic Appl. Sci., 3, 263 (2014).

14. P. Alexy, D. Kachova, M. Krsiak, D. Bakos and B. Simkova, Polym. Degrad. Stab., 78, 413 (2002);

https://doi.org/10.1016/S0141-3910(02)00177-5.

15. J. Shin and S.E.M. Selke, in eds.: S. Clark, S. Jung and B. Lamsal, Food Processing: Principles and Applications, John Wiley \& Sons, edn 2, Chap. 11, p. 249 (2014).

16. P. Calvo, C. Remunan-Lopez, J.L. Vila-jato and M.J. Alonso, J. Appl. Polym. Sci., 63, 125 (1997); https://doi.org/10.1002/(SICI)1097-4628(19970103)63:1<125::AIDAPP13>3.0.CO;2-4.
17. K. Nagpal, S.K. Singh and D.N. Mishra, Chem. Pharm. Bull. (Tokyo), 58, 1423 (2010); https://doi.org/10.1248/cpb.58.1423.

18. E.M.S. Cordeiro, Y.L. Nunes, A.L.A. Mattos, M.F. Rosa, M. de sá M. Sousa Filho and E.N. Ito, Macromol. Symp., 344, 39 (2014); https://doi.org/10.1002/masy.201300217.

19. M.F. Rosa, E.S. Medeiros, J.A. Malmonge, K.S. Gregorski, D.F. Wood, L.H.C. Mattoso, G. Glenn, W.J. Orts and S.H. Imam, Carbohydr. Polym., 81, 83 (2010); https://doi.org/10.1016/j.carbpol.2010.01.059.

20. E.D. Cranston and D.G. Gray, Biomacromolecules, 7, 2522 (2006); https://doi.org/10.1021/bm0602886.

21. S.R. Muppalla, S.R. Kanatt, S. Chawla and A. Sharma, Food Packag. Shelf Life, 2, 51 (2014); https://doi.org/10.1016/j.fps1.2014.07.002.

22. I.M. Thakore, S. Desai, B.D. Sarawade and S. Devi, Eur. Polym. J., 37, 151 (2001); https://doi.org/10.1016/S0014-3057(00)00086-0.

23. D. Prajakta, J. Ratnesh, K. Chandan, S. Suresh, S. Grace, V. Meera and P. Vandana, J. Biomed. Nanotechnol., 5, 445 (2009); https://doi.org/10.1166/ibn.2009.1038.

24. S.Azizi, M. Ahmad, M. Mahdavi and S. Abdolmohammadi, BioResources, 8, 1841 (2013); https://doi.org/10.15376/biores.8.2.1841-1851.

25. H.Z. Li, S.C. Chen and Y.Z. Wang, Compos. Sci. Technol., 115, 60 (2015); https://doi.org/10.1016/j.compscitech.2015.05.004.

26. T. Gomathi, C. Govindarajan, M.H. Rose, P.N. Sudha, P.K.M. Imran, J. Venkatesan and S.-K. Kim, Int. J. Pharm., 468, 214 (2014); https://doi.org/10.1016/j.ijpharm.2014.04.026. 